\title{
Für schwangere Angestellte gelten besondere Regelungen
}

\author{
Für werdende und stillende Mütter, die in einem Beschäftigungsverhältnis stehen, hat der \\ Gesetzgeber eine Reihe von Bestimmungen erlassen, um das Leben und die Gesundheit von \\ Mutter und Kind zu schützen. Diese finden sich insbesondere im Mutterschutzgesetz (MuSchuG) \\ und in der Verordnung zum Schutze der Mütter am Arbeitsplatz (MuSchArbV). Darüber sollten \\ Zahnärzte als Arbeitgeber genauso informiert sein wie werdende Mütter.
}

Damit der Arbeitgeber die Vorschriften einhalten kann, soll die werdende Mutter ihn möglichst schnell über die Schwangerschaft und den voraussichtlichen Entbindungstermin informieren. Danach erfolgt eine Meldung an die zuständige Aufsichtsbehörde, in der über Arbeitszeit und Art der Tätigkeit der Schwangeren informiert wird, die sogenannte Schwangerschaftsanzeige. Da Arbeitnehmerinnen in einer Zahnarztpraxis während der Schwangerschaft und Stillzeit einer Reihe von Gefährdungen ausgesetzt sind, ist eine Gefährdungsbeurteilung $(\$ 1 \mathrm{MuSch} A r b V)$ des jeweiligen Arbeitsplatzes vorzunehmen. Die Beurteilung erstreckt sich auf jede Tätigkeit, die die werdende oder stillende Mutter durchführt und beinhaltet Art, Ausmaß und Dauer der Gefährdung. Von besonderer Bedeutung im medizinischen Bereich ist dabei der Umgang mit strahlendem, toxischem oder infektiösem Material.

\section{Geeignete Schutzmaßnahmen ergreifen}

Ergibt die Analyse eine Gefährdung von Mutter oder Kind muss der Zahnarzt geeignete Schutzmaßnahmen ( $\$ 3 \mathrm{MuSchArbV)}$ veranlassen. Diese können in der Umgestaltung des Arbeitsplatzes, im Arbeitsplatzwechsel oder aber auch in der Freistellung wegen eines Beschäftigungsverbots liegen. Alle Angestellten der Zahnarztpraxis müssen über die Ergebnisse und die zu treffenden Schutzmaßnahmen informiert werden.

Insbesondere liegen folgende Beschäftigungsverbote oder -einschränkungen vor:

1. Individuelles Beschäftigungsverbot mit ärztlichem Attest;

2. Arbeitsverbot zwischen 20:00 und 6:00;

3. Verbot der Mehrarbeit (Arbeitszeiten von mehr als 8,5 Stunden pro Tag beziehungsweise 90 Stunden in der Doppelwoche);

4. Verbot der Sonn- und Feiertagsarbeit;

5. Verbot der Beschäftigung mit sehr giftigen, giftigen, gesundheitsschädlichen oder in sonstiger Weise den Menschen chronisch schädigenden Gefahrstoffen, wenn der Arbeitsplatzgrenzwert (AWG) überschritten wird (Hinweise auf Produktverpackungen und Sicherheitsdatenblättern; Beispiele: Desinfektions- und Reinigungsmittel, Lösemittel, Röntgenchemikalien, Quecksilber);

6. Verbot der Beschäftigung mit Stoffen, Zubereitungen und Erzeugnissen, die ihrer Art nach erfahrungsgemäß Krankheitserreger übertragen können, zum Beispiel Blut, Speichel, Aerosole, die bei der Behandlung entstehen;

7. Einhaltung der Grenzwerte nach Röntgenverordnung(RöV) beim Arbeiten an Röntgeneinrichtungen.

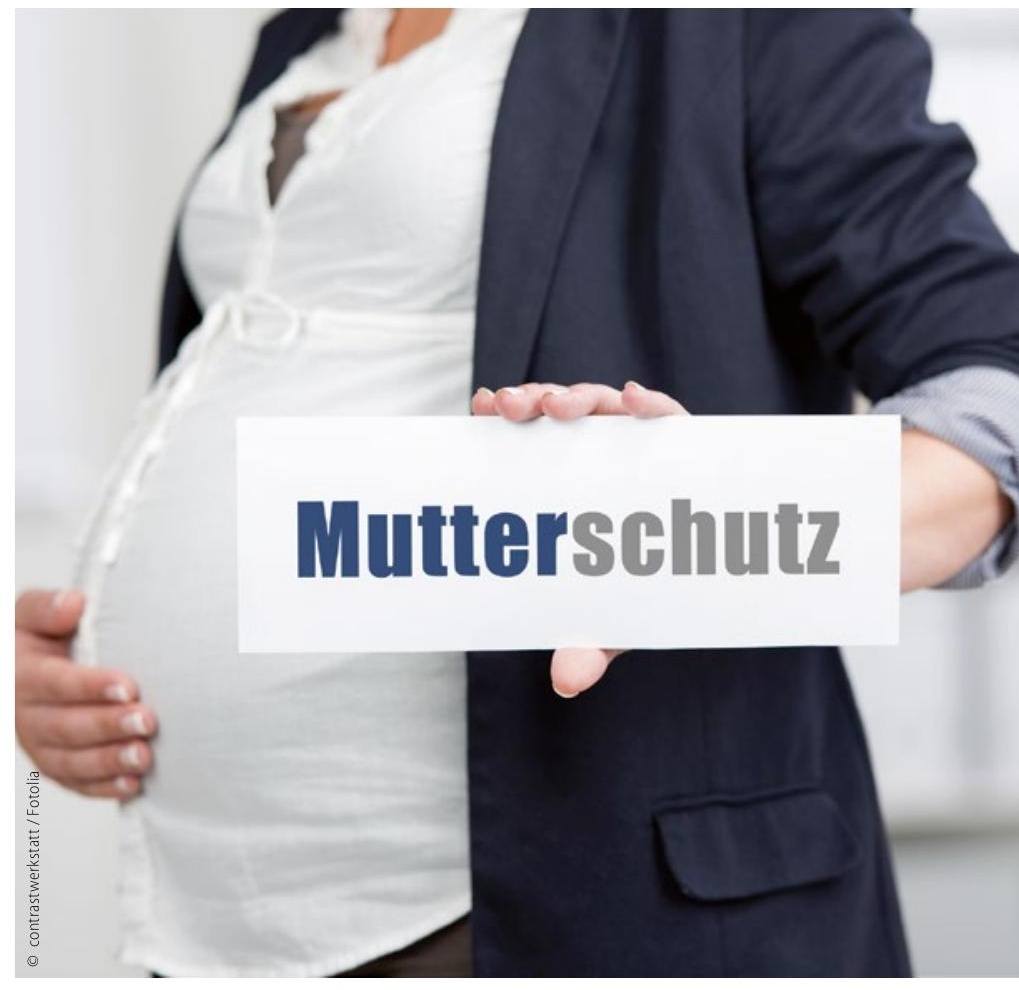

Aufgrund der genannten Einschränkungen ist der Arbeitsplatz einer schwangeren Angestellten besser in den administrativen Teil der Zahnarztpraxis, beispielsweise an den Empfangstresen, zu verlegen. Wenn dies in der Praxis nicht möglich ist, dann ist gegebenenfalls ein Beschäftigungsverbot auszusprechen. Für eine angestellte Zahnärztin, deren Hauptaufgaben darin liegen, Patienten zu behandeln, wird eine Schwangerschaft meist auf ein Beschäftigungsverbot hinauslaufen.

Eine Kündigung ist während der Schwangerschaft und in der Schutzfrist von vier Monaten nach der Entbindung nicht möglich. Diese Schutzbedingungen gelten für alle angestellten Mitarbeiterinnen einer Praxis, Praxisinhaberinnen dürfen jedoch während der eigenen Schwangerschaft und Stillzeit weiterhin arbeiten.

Dr. Judith Schimann, Zahnärztin und freie Journalistin

Quelle: Wir in der Praxis, 1/2016 